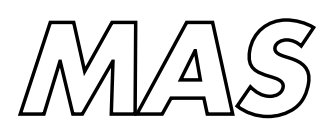

Modelling, Analysis and Simulation

Modelling, Analysis and Simulation
MAS computing solutions of the modified Bessel differential
equation for imaginary orders and positive arguments

A. Gil, J. Segura, N.M. Temme

Report MAS-E0416 October 2004 
CWI is the National Research Institute for Mathematics and Computer Science. It is sponsored by the Netherlands Organization for Scientific Research (NWO).

CWI is a founding member of ERCIM, the European Research Consortium for Informatics and Mathematics.

CWI's research has a theme-oriented structure and is grouped into four clusters. Listed below are the names of the clusters and in parentheses their acronyms.

Probability, Networks and Algorithms (PNA)

Software Engineering (SEN)

Modelling, Analysis and Simulation (MAS)

Information Systems (INS)

Copyright (C) 2004, Stichting Centrum voor Wiskunde en Informatica

P.O. Box 94079, 1090 GB Amsterdam (NL)

Kruislaan 413, 1098 SJ Amsterdam (NL)

Telephone +31205929333

Telefax +31205924199

ISSN 1386-3703 


\title{
Computing solutions of the modified Bessel differential equation for imaginary orders and positive arguments
}

\author{
ABSTRACT \\ implementation of these methods in Fortran 77 codes. \\ 2000 Mathematics Subject Classification: 65D20, 33C10, 41A60 \\ 1998 ACM Computing Classification System: G.4 \\ Keywords and Phrases: Bessel functions, numerical quadrature, asymptotic expansions \\ Note: Accepted for publication in ACM Transactions on Mathematical Software
}

We describe a variety of methods to compute the functions $K_{i a}(x), L_{i a}(x)$ and their derivatives for real $a$ and positive $x$. These functions are numerically satisfactory independent solutions of the differential equation $x^{2} w^{\prime \prime}+x w^{\prime}+\left(a^{2}-x^{2}\right) w=0$. In an accompanying paper, we describe the 



\title{
Computing solutions of the modified Bessel differential equation for imaginary orders and positive arguments
}

\author{
Amparo Gil ${ }^{1}$ \\ Departamento de Matemáticas, U. Autónoma de Madrid, 28049-Madrid, Spain \\ Javier Segura \\ Departamento de Matemáticas, Estadística y Computación. U. de Cantabria, 39005- \\ Santander, Spain \\ and \\ Nico M. Temme \\ CWI, P.O. Box 94079, 1090 GB Amsterdam, The Netherlands
}

\begin{abstract}
We describe a variety of methods to compute the functions $K_{i a}(x), L_{i a}(x)$ and their derivatives for real $a$ and positive $x$. These functions are numerically satisfactory independent solutions of the differential equation $x^{2} w^{\prime \prime}+x w^{\prime}+\left(a^{2}-x^{2}\right) w=0$. In the accompanying paper [6], we describe the implementation of these methods in Fortran 77 codes.

Categories and Subject Descriptors: G.4 [Mathematics of Computing]: Mathematical software General Terms: Algorithms
\end{abstract}

Additional Key Words and Phrases: Bessel functions, numerical quadrature, asymptotic expansions

\section{INTRODUCTION}

In previous publications $[7 ; 8]$, methods to compute the modified Bessel function $K_{i a}(x)$ for positive $x$ were described. We complete here this analysis by describing analogous methods for the computation of the function $L_{i a}(x)$. With this, methods for the reliable computation of a pair of linearly independent numerically satisfactory solutions become available which find their implementation in the accompanying paper [6]. Methods to compute their derivatives are also provided.

The functions $K_{i a}(x)$ and $L_{i a}(x)$ are solutions of the modified Bessel equation for imaginary orders

$$
x^{2} w^{\prime \prime}+x w^{\prime}+\left(a^{2}-x^{2}\right) w=0
$$

${ }^{1}$ Present address: Departamento de Matemáticas, Estadística y Computación. U. de Cantabria, 39005-Santander, Spain 
The function $K_{i a}(x)$ finds application in a number of problems of physics and applied mathematics [7]. The function $L_{i a}(x)$ is a real valued numerically satisfactory companion to $K_{i a}(x)$ in the sense described in [11], pp. 154-155.

In terms of the modified Bessel function of the first kind $I_{\nu}(x)$, the solutions are defined as:

$$
K_{i a}(x)=\frac{\pi}{2 i \sinh (\pi a)}\left[I_{-i a}(x)-I_{i a}(x)\right], L_{i a}(x)=\frac{1}{2}\left[I_{-i a}(x)+I_{i a}(x)\right],
$$

with Wronskian $W\left[K_{i a}(x), L_{i a}(x)\right]=1 / x$.

Both $K_{i a}(x)$ and $L_{i a}(x)$ are real solutions for real $x>0$ and $a \in \mathbb{R}$. Because they are even functions of $a$, in the sequel we will consider $a \geq 0$, although this restriction is not present in the code.

In Section 2, we describe the different methods of computation considered, namely: series expansions, asymptotic expansions for large $x$, Airy-type uniform asymptotic expansions, non-oscillating integral representations (including a discussion on the quadrature rule) and a continued fraction method. We avoid duplicating information already given in previous papers; in particular, the references $[7 ; 8]$ provide information required for building the algorithms of the accompanying paper [6]. A few misprints in [7] are corrected.

In Section 3, we include a discussion on the dominant asymptotic behaviour of the functions. These exponential dominant factors can be taken out, leading to scaled functions which can be computed in a much wider range. The algorithm described in the accompanying paper [6] offers the possibility of computing scaled and unscaled functions.

\section{METHODS OF COMPUTATION}

In $[7 ; 8]$ methods are described to compute the $K_{i a}(x)$ for different regions in the $(x, a)$ plane. In particular, we considered series expansions [16], asymptotic expansions for large $x$ ([1], Eq. 9.7.2), uniform asymptotic expansions for $a \simeq x$ $([2 ; 4]$ and $[11]$, pg. 425). Also, non-oscillating integral representations [17; 7] are available. Similar techniques are available for the computation of $L_{i a}(x)$ and the derivatives $K_{i a}^{\prime}(x), L_{i a}^{\prime}(x)$. In addition, a continued fraction method can be applied for the computation of $K_{i a}(x)$ and $K_{i a}^{\prime}(x)$. Those techniques generally give at least two alternative methods for computing the functions in the $(x, a)$ plane for moderate values of $x$ and $a$; therefore, we can always compare different methods of computation for testing their accuracy. The selection of one or another method of computation in a given region will depend on the range of applicability of each method and its efficiency.

We now describe the different methods of computation which are used in the programs.

\subsection{Series expansions}

Series expansions can be built which properly describe the solutions near the singular point $(x=0)$ of the defining differential equation (1). The idea, as described in $[16 ; 7]$, is to substitute the Maclaurin series for $I_{\nu}(x)([1]$, Eq. 9.6.10) in Eqs. (2). The following expansions are obtained 


$$
\begin{aligned}
& K_{i a}(x)=\frac{1}{n(a)} \sum_{k=0}^{\infty} f_{k} c_{k}, K_{i a}^{\prime}(x)=\frac{1}{n(a)} \frac{2}{x} \sum_{k=0}^{\infty}\left[k f_{k}-\frac{r_{k}}{2}\right] c_{k} \\
& L_{i a}(x)=n(a) \sum_{k=0}^{\infty} r_{k} c_{k} \quad, L_{i a}^{\prime}(x)=n(a) \frac{2}{x} \sum_{k=0}^{\infty}\left[k r_{k}+a^{2} \frac{f_{k}}{2}\right] c_{k},
\end{aligned}
$$

where

$$
n(a)=e^{\pi a / 2} \sqrt{\frac{1-e^{-2 \pi a}}{2 \pi a}}, \quad c_{k}=(x / 2)^{2 k} / k !
$$

and $[4]$

$$
\begin{aligned}
& f_{k}=\frac{\sin \left(\phi_{a, k}-a \ln (x / 2)\right)}{\left(a^{2}\left(1+a^{2}\right) \ldots\left(k^{2}+a^{2}\right)\right)^{1 / 2}}, \\
& f_{k} / r_{k}=\frac{1}{a} \tan \left(\phi_{a, k}-a \ln (x / 2)\right), \text { with } \phi_{a, k}=\arg (\Gamma(1+k+i a)) .
\end{aligned}
$$

The coefficients $f_{k}$ and $r_{k}$ differ from those in [7] by a constant factor (for fixed a). The new normalization shows explicitly (Eqs. (3)) the dominant exponential behaviour $n(a)$ and $1 / n(a)$ as $a \rightarrow \infty\left(\sim e^{ \pm \pi a / 2}\right)$.

An efficient method to compute the coefficients was described in $[7 ; 16]$; this method is based on the fact that both $f_{k}$ and $r_{k}$ satisfy the three-term recurrence relation

$$
\left(k^{2}+a^{2}\right) r_{k}-(2 k-1) r_{k-1}+r_{k-2}=0 .
$$

Perron's theorem is inconclusive with respect to the existence of minimal solutions for this recurrence relation; anyhow, the second equation in (5) confirms that neither $f_{k}$ nor $r_{k}$ are minimal solutions. Therefore, forward recursion will be numerically stable. Starting values can be computed taking into account that arg $\Gamma(1+i a)=$ $\sigma_{0}(a)$, where $\sigma_{0}(a)$ is the Coulomb phase shift, for which Chebyshev expansions are available for double precision [3]. Namely, we have:

$$
\begin{aligned}
& r_{0}=\cos \left[\sigma_{0}(a)-a \ln (x / 2)\right], \\
& r_{1}=\frac{1}{1+a^{2}}\left\{\cos \left[\sigma_{0}(a)-a \ln (x / 2)\right]-a \sin \left[\sigma_{0}(a)-a \ln (x / 2)\right]\right\},
\end{aligned}
$$

and

$$
\begin{aligned}
& f_{0}=\frac{1}{a} \sin \left[\sigma_{0}(a)-a \ln (x / 2)\right], \\
& f_{1}=\frac{1}{a\left(1+a^{2}\right)}\left\{\sin \left[\sigma_{0}(a)-a \ln (x / 2)\right]+a \cos \left[\sigma_{0}(a)-a \ln (x / 2)\right]\right\} .
\end{aligned}
$$

These formulas correct two misprints in [7] (Eqs. 12 and 13).

Series can be used for $x / a$ small. See [6], Section 2 . 


\subsection{Asymptotic expansions for large $x$}

Asymptotic expansions for large $x$ are available from the known asymptotic expansion of $I_{\nu}(x)([1]$, Eq. 9.7.1):

$$
\begin{aligned}
& K_{i a}(x)=\left(\frac{\pi}{2 x}\right)^{1 / 2} e^{-x}\left\{\sum_{k=0}^{n-1} \frac{(i a, k)}{(2 x)^{k}}+\gamma_{n}\right\}, \\
& L_{i a}(x)=\frac{1}{\sqrt{2 \pi x}} e^{x}\left\{\sum_{k=0}^{n-1}(-1)^{k} \frac{(i a, k)}{(2 x)^{k}}+\delta_{n}\right\},
\end{aligned}
$$

where $(i a, m)$ is the Hankel symbol, which satisfies

$$
(i a, k+1)=-\frac{\left(k+\frac{1}{2}\right)^{2}+a^{2}}{k+1}(i a, k),(i a, 0)=1 .
$$

Bounds for the error terms $\left(\gamma_{n}, \delta_{n}\right)$ can be found in [11], Pg. 269, Ex. 13.2.

As discussed in [7] the numerical performance of the asymptotic expansion for $K_{i a}(x)$ is of more restricted applicability than for the case of the evaluation of $K_{\nu}(x)$ for real $\nu$. Furthermore, the continued fraction method described in [7] covers the region where this expansion is of numerical interest. For this reason, the continued fraction method is the preferred algorithm for the evaluation of $K_{i a}(x)$ and $K_{i a}^{\prime}(x)$ for moderate values of $a$. On the other hand the asymptotic expansion for $L_{i a}(x)$ turns out to be accurate in a wider region, which is a fortunate situation given that the continued fraction method is not available in this case. See [6], Section 2, for further details.

Asymptotic expansions for the derivatives are also available by differentiating Eqs. (9).

\subsection{Airy-type uniform asymptotic expansions}

The Airy-type asymptotic expansions for $K_{i a}(x)$ can be found in [2; 4] and [11] pg. 425; the analogous expansions for $L_{i a}(x)$ [4] and $K_{i a}^{\prime}(x)$ are also available [2], while the expansion for $L_{i a}^{\prime}(x)$ can be derived in the same way. We summarize here the main features needed for the computation through these expansions, neglecting the error terms. Further details can be found in $[2 ; 4 ; 11]$ and $[8 ; 18]$.

The expansion for $K_{i a}(x)$ and $L_{i a}(x)$ in terms of Airy functions $(\operatorname{Ai}(z), \operatorname{Bi}(z)$ and their derivatives) reads

$$
\begin{aligned}
& K_{i a}(a z)=\frac{\pi e^{-a \pi / 2} \phi(\zeta)}{a^{1 / 3}}\left[\operatorname{Ai}\left(-a^{2 / 3} \zeta\right) F_{a}(\zeta)+\frac{1}{a^{4 / 3}} \operatorname{Ai}^{\prime}\left(-a^{2 / 3} \zeta\right) G_{a}(\zeta)\right], \\
& L_{i a}(a z)=\frac{e^{a \pi / 2} \phi(\zeta)}{2 a^{1 / 3}}\left[\operatorname{Bi}\left(-a^{2 / 3} \zeta\right) F_{a}(\zeta)+\frac{1}{a^{4 / 3}} \operatorname{Bi}^{\prime}\left(-a^{2 / 3} \zeta\right) G_{a}(\zeta)\right],
\end{aligned}
$$

where

$$
F_{a}(\zeta) \sim \sum_{s=0}^{\infty}(-)^{s} \frac{a_{s}(\zeta)}{a^{2 s}}, G_{a}(\zeta) \sim \sum_{s=0}^{\infty}(-)^{s} \frac{b_{s}(\zeta)}{a^{2 s}}
$$


as $a \rightarrow \infty$ uniformly with respect to $z \in[0, \infty)$. Error bounds for the asymptotic expansions of the $K_{i a}(x)$ and $L_{i a}(x)$ are given in [4].

The quantity $\zeta$ is given by

$$
\begin{aligned}
& \frac{2}{3} \zeta^{3 / 2}=\log \frac{1+\sqrt{1-z^{2}}}{z}-\sqrt{1-z^{2}}, 0 \leq z \leq 1, \\
& \frac{2}{3}(-\zeta)^{3 / 2}=\sqrt{z^{2}-1}-\arccos \frac{1}{z}, z \geq 1,
\end{aligned}
$$

and

$$
\phi(\zeta)=\left(\frac{4 \zeta}{1-z^{2}}\right)^{1 / 4}, \phi(0)=2^{1 / 3} .
$$

Of course, it is crucial to compute accurately Eqs. (13) for small $\zeta$. For this, series expansions around $z=1$ can be used.

The evaluation of the coefficients near the turning point $z=1$ (which is our region of interest) can be performed via Maclaurin series expansions of the quantities $\phi$, $a_{s}$ and $b_{s}([18])$ in terms of the variable $\eta=2^{-1 / 3} \zeta$ (see [8] and [18] for further details).

Asymptotic expansions for the derivatives can be found by differentiating Eqs. (11). In this way:

$$
\begin{aligned}
& K_{i a}^{\prime}(a z)=2 \frac{\pi e^{-a \pi / 2}}{z a^{2 / 3} \phi(\zeta)}\left[\operatorname{Ai}^{\prime}\left(-a^{2 / 3} \zeta\right) P_{a}(\zeta)+\frac{1}{a^{2 / 3}} \operatorname{Ai}\left(-a^{2 / 3} \zeta\right) Q_{a}(\zeta)\right] \\
& L_{i a}^{\prime}(a z)=\frac{e^{a \pi / 2}}{z a^{2 / 3} \phi(\zeta)}\left[\operatorname{Bi}^{\prime}\left(-a^{2 / 3} \zeta\right) P_{a}(\zeta)+\frac{1}{a^{2 / 3}} \operatorname{Bi}\left(-a^{2 / 3} \zeta\right) Q_{a}(\zeta)\right]
\end{aligned}
$$

where $P_{a}(\zeta)$ and $Q_{a}(\zeta)$ can be written in terms of $F_{a}(\zeta), G_{a}(\zeta)$ and their derivatives and they have asymptotic expansions

$$
P_{a}(\zeta) \sim \sum_{s=0}^{\infty}(-)^{s} \frac{c_{s}(\zeta)}{a^{2 s}}, Q_{a}(\zeta) \sim \sum_{s=0}^{\infty}(-)^{s} \frac{d_{s}(\zeta)}{a^{2 s}},
$$

whose coefficients can be obtained from the computed coefficients $a_{s}$ and $b_{s}$ (in Taylor series around $\zeta=0$ ) through the relations:

$$
\begin{aligned}
& c_{s}(\zeta)=a_{s}(\zeta)+\chi(\zeta) b_{s-1}(\zeta)+b_{s-1}^{\prime}(\zeta), \\
& d_{s}(\zeta)=-\chi(\zeta) a_{s}(\zeta)-a_{s}^{\prime}(\zeta)-\zeta b_{s}(\zeta),
\end{aligned}
$$

where

$$
\chi(\zeta)=\phi^{\prime}(\zeta) / \phi(\zeta)
$$

The prime in Eqs. (17) and (18) denotes the derivative with respect to $\zeta$. Using Eqs. (17) the coefficients $c_{s}$ and $d_{s}$ can be computed from the coefficients $a_{s}$ and $b_{s}$. Details on the evaluation of $a_{s}$ and $b_{s}$ are given in [8], where an explicit Maple algorithm is given for the computation of $a_{s}$ and $b_{s}$ for $s=0,1,2,3$. 
By computing the Wronskian relation for the modified Bessel functions and using the Wronskian for Bessel functions, it is easy to derive the relation

$$
F_{a}(\zeta) P_{a}(\zeta)-\frac{1}{a^{2}} G_{a}(\zeta) Q_{a}(\zeta)=1
$$

which is a useful relation for checking the correctness of the approximations for the coefficients in the asymptotic expansions.

An algorithm to compute Airy functions of a real variable is needed for the computation of these asymptotic expansions. In the routines [6] we use Algorithm $819[9]$.

These Airy-type asymptotic expansion are applied in [6] in a broad region around the turning point line $a=x$.

\subsection{Non-oscillating integral representations}

Paths of steepest descent for integral representations of the modified Bessel functions of imaginary orders and their derivatives are given in [17]. Apart from their application in asymptotics [5], these integrals are useful for building numerically stable (non-oscillating) integral representations for $K_{i a}(x)$ and $K_{i a}^{\prime}(x)$, as described in [7]. We complete here the analysis in [7] by providing analogous expressions for the computation of $L_{i a}(x)$ and $L_{i a}^{\prime}(x)$. Additionally, we study further transformations of the integrals which enable us to obtain integral expressions suitable for computation by means of the trapezoidal rule.

2.4.1 Monotonic case $(x>a)$. We have the following integral representations in the monotonic region $[7]$

$$
\begin{aligned}
& K_{i a}(x)=e^{-\lambda} \int_{0}^{\infty} e^{-x \Phi(\tau)} d \tau \\
& K_{i a}^{\prime}(x)=-e^{-\lambda} \int_{0}^{\infty}\left[\cos \theta+\frac{\cosh \tau-1+2 \sin ^{2} \frac{1}{2}(\theta-\sigma)}{\cos \sigma}\right] e^{-x \Phi(\tau)} d \tau,
\end{aligned}
$$

where

$$
\lambda=x \cos \theta+a \theta, a=x \sin \theta, \sin \sigma=\left(\sin \theta \frac{\tau}{\sinh \tau}\right)
$$

and $\theta \in[0, \pi / 2), \sigma \in(0, \theta]$. The dominant exponential term $\left(e^{-\lambda}\right)$ has been factored out. The argument of the exponential in the integrand is

$$
\Phi(\tau)=(\cosh \tau-1) \cos \sigma+2 \sin \left(\frac{\theta-\sigma}{2}\right) \sin \left(\frac{\theta+\sigma}{2}\right)+(\sigma-\theta) \sin \theta
$$

This formula corrects a misprint in [7] (Eq. 33). The difference $\theta-\sigma$ can be computed in a stable way for small values of $\tau$ by using the expression.

$$
\sin (\theta-\sigma)=\frac{\sin \theta}{\cos \theta \frac{\tau}{\sinh \tau}+\cos \sigma}\left[1-\frac{\tau^{2}}{\sinh ^{2} \tau}\right]
$$

together with the definition of $\sigma(21)$ and specific algorithms to compute $\cosh (\tau)-1$ and $1-\sinh (\tau)^{2} / \tau^{2}$ for small $\tau$. 
The non-oscillating integral representations for $L_{i a}(x)$ and its derivative can be written after factoring the dominant exponential contribution as:

$$
L_{i a}(x)=\frac{e^{\lambda}}{2 \pi}\left[\int_{-\theta-\pi}^{-\theta+\pi} e^{x \gamma(\sigma)} d \sigma-\left(1-e^{-2 \pi a}\right) e^{-\eta} \int_{0}^{+\infty} e^{-x \Phi(\tau)} \frac{d \sigma}{d \tau} d \tau\right]
$$

where

$$
\begin{aligned}
& \gamma(\sigma)=2 \sin \frac{\theta-\sigma}{2} \sin \frac{\theta+\sigma}{2}+(\sigma-\theta) \sin \theta \\
& \eta=2 x[\cos \theta+(\theta-\pi / 2) \sin \theta]=2 x\left(\sqrt{1-(a / x)^{2}}-\frac{a}{x} \arccos \left(\frac{a}{x}\right)\right)
\end{aligned}
$$

and using Eq. (21)

$$
\frac{d \sigma}{d \tau}=\tan \sigma\left[\frac{1}{\tau}-\operatorname{coth} \tau\right]
$$

The first integral is dominant over the second one for large values of the parameters and $a / x$ not too close to $a=x$. As $a \rightarrow x$ both integrals become of the same order.

Similarly, we have the following representation for $L_{i a}^{\prime}(x)$ :

$$
L_{i a}^{\prime}(x)=\frac{e^{\lambda}}{2 \pi}\left[\int_{-\theta-\pi}^{-\theta+\pi} \cos \sigma e^{x \gamma(\sigma)} d \sigma+\left(1-e^{-2 \pi a}\right) e^{-\eta} \int_{0}^{+\infty} e^{-x \Phi(\tau)} h(\tau) d \tau\right]
$$

where

$$
h(\tau)=\sin \sigma\left[\frac{\cosh \tau}{\tau}-\frac{1}{\sinh \tau}\right]
$$

These integral representations for $L_{i a}(x)$ and $L_{i a}^{\prime}(x)$ can be used for checking the computation of these functions in the monotonic region. They are not used by our algorithms [6] because the Airy-type asymptotic expansion (Section 2.3) and the expansion for large $x$ (Section 2.2) are sufficiently accurate for this functions and they are faster to compute (see [6], Section 2).

2.4.2 Oscillatory case $(x<a)$. The non-oscillating integral representations for the oscillatory region are more difficult to evaluate numerically than those for the monotonic case. Indeed, as it was discussed in [7], the steepest descent method leads to three integrals, which have to be computed separately. However, as we later discuss, for moderately large $a$ it will be enough to compute a single integral.

In [7], the following formula was obtained: 


$$
\begin{aligned}
K_{i a}(x) & =e^{-\pi a / 2}\left[\int_{\mu}^{\infty} e^{-\Psi(\tau)}\left(\cos \chi+\sin \chi \frac{d \sigma}{d \tau}\right) d \tau\right. \\
& +\frac{1}{\sinh \pi a} \int_{\mu-\tanh \mu}^{\mu}\left(\cos \chi \sinh \rho+\sin \chi \cosh \rho \frac{d \sigma}{d \tau}\right) d \tau \\
& \left.-\frac{1}{\sinh \pi a} \int_{\pi}^{3 \pi / 2}\left(\cos \chi \sinh \rho \frac{d \tau}{d \sigma}+\sin \chi \cosh \rho\right) d \sigma\right],
\end{aligned}
$$

where $\chi=x \sinh \mu-a \mu, \cosh \mu=\frac{a}{x}, \mu>0$,

$$
\Psi(\tau)=x \cosh \tau \cos \sigma+a\left(\sigma-\frac{1}{2} \pi\right), \rho(\tau)=-\Psi(\tau)+a \pi
$$

and

$$
\sin \sigma=\frac{(\tau-\mu) \cosh \mu+\sinh \mu}{\sinh \tau} .
$$

Notice that each of the three integrals in Eq. (29) can in principle be integrated with respect to any of the two variables $\sigma$ and $\tau$, taking into account Eq. (31) together with the fact that the integration path $\tau(\sigma)$ is such that $\tau(0)=+\infty$, $\tau(\pi / 2)=\mu, \tau(\pi)=\mu-\tanh \mu>\tau(3 \pi / 2)$; however, as discussed in [7] there are strong numerical reasons for the selections made. In particular, the third integral is performed with respect to $\sigma$ (which requires numerical inversion of (31)) to avoid the singularity of $d \sigma / d \tau$ at $\tau(3 \pi / 2)$. As explained in [7] the numerical inversion of (31) in the interval $\sigma \in[\pi, 3 \pi / 2]$ can be efficiently performed in parallel with the numerical integration.

Similar integral representations exist for $K_{i a}^{\prime}(x), L_{i a}(x)$ and $L_{i a}^{\prime}(x)$. We have:

$$
\begin{aligned}
K_{i a}^{\prime}(x) & =e^{-\pi a / 2}\left[\int_{\mu}^{\infty} e^{-\Psi(\tau)}(\cos \chi A(\tau)+\sin \chi C(\tau)) d \tau\right. \\
& +\frac{1}{\sinh \pi a} \int_{\mu-\tanh \mu}^{\mu}(\cos \chi \cosh \rho A(\tau)+\sin \chi \sinh \rho C(\tau)) d \tau \\
& \left.-\frac{1}{\sinh \pi a} \int_{\pi}^{3 \pi / 2}(\cos \chi \cosh \rho B(\tau(\sigma))+\sin \chi \sinh \rho D(\tau(\sigma))) d \sigma\right]
\end{aligned}
$$

where

$$
\begin{aligned}
& A(\tau)=-\cosh \tau \cos \sigma+\sinh \tau \sin \sigma \frac{d \sigma}{d \tau}, B(\tau)=A(\tau) \frac{d \tau}{d \sigma} \\
& C(\tau)=-\sinh \tau \sin \sigma-\cosh \tau \cos \sigma \frac{d \sigma}{d \tau}, D(\tau)=C(\tau) \frac{d \tau}{d \sigma}
\end{aligned}
$$

In addition, integral representations for $L_{i a}(x)$ and its derivative are: 


$$
\begin{aligned}
L_{i a}(x) & =\frac{e^{\pi a / 2}}{\pi}\left[\frac{1-e^{-2 \pi a}}{2} \int_{\mu}^{\infty} e^{-\Psi(\tau)}\left(\sin \chi-\cos \chi \frac{d \sigma}{d \tau}\right) d \tau\right. \\
& +e^{-\pi a} \int_{\mu-\tanh \mu}^{\mu}\left(\sin \chi \sinh \rho-\cos \chi \cosh \rho \frac{d \sigma}{d \tau}\right) d \tau \\
& \left.-e^{-\pi a} \int_{\pi}^{3 \pi / 2}\left(\sin \chi \sinh \rho \frac{d \tau}{d \sigma}-\cos \chi \cosh \rho\right) d \sigma\right]
\end{aligned}
$$

and

$$
\begin{aligned}
L_{i a}^{\prime}(x) & =\frac{e^{\pi a / 2}}{\pi}\left[\frac{1-e^{-2 \pi a}}{2} \int_{\mu}^{\infty} e^{-\Psi(\tau)}(\sin \chi A(\tau)-\cos \chi C(\tau)) d \tau\right. \\
& +e^{-\pi a} \int_{\mu-\tanh \mu}^{\mu}(\sin \chi \cosh \rho A(\tau)-\cos \chi \sinh \rho C(\tau)) d \tau \\
& \left.-e^{-\pi a} \int_{\pi}^{3 \pi / 2}(\sin \chi \cosh \rho B(\tau(\sigma))-\cos \chi \sinh \rho D(\tau(\sigma))) d \sigma\right]
\end{aligned}
$$

Notice that the dominant exponential behaviour has been factored for both the functions $K_{i a}(x)$ and $L_{i a}(x)$ and their derivatives, which coincides with the exponential behaviour of the uniform asymptotic expansion. This is an interesting feature when computing scaled functions in order to avoid overflows and/or underflows in the computation. After factoring the dominant exponential terms $\left(e^{ \pm \pi a / 2}\right)$, the overflow and/or underflow problems are eliminated; notice, however, that when computing the integrals over finite intervals we should evaluate $\sinh (\rho) / e^{a \pi}, \cosh (\rho) / e^{a \pi}$ for $L_{i a}(x)$ and its derivative and $\sinh (\rho) / \sinh a \pi$, $\cosh (\rho) / \sinh a \pi$ for $K_{i a}(x)$ (and $K_{i a}^{\prime}(x)$ ) instead of computing the hyperbolic and the exponential separately (otherwise, overflows will take place for moderately large a). For this reason it is convenient to use the expressions

$$
\frac{\cosh \rho}{\sinh \pi a}=e^{-\psi} \frac{1+e^{-2 \rho}}{1-e^{-2 \pi a}}, \frac{\sinh \rho}{\sinh \pi a}=e^{-\psi} \frac{1-e^{-2 \rho}}{1-e^{-2 \pi a}}
$$

in the evaluation of Eqs. (29) and (32) and to proceed in the same way for $e^{-\pi a} \cosh \rho$ and $e^{-\pi a} \sinh \rho$ in Eqs. (34) and (35). Notice that in the oscillatory region $\rho>0$ and that for large $a$ and $x$ both $e^{-2 \rho}$ and $e^{-2 \pi a}$ will underflow. These underflow problems can be easily avoided by neglecting these exponential terms for large parameters.

In addition, when both exponentials become negligible, the integral over sigma becomes negligible and the remaining two integrals can be approximated by only one integral. We can write 


$$
\begin{aligned}
& K_{i a}(x) \approx e^{-\pi a / 2}\left[\int_{\tau_{0}}^{\infty} e^{-\Psi(\tau)}\left(\cos \chi+\sin \chi \frac{d \sigma}{d \tau}\right) d \tau+\mathcal{O}\left(e^{-\pi a / 2}\right)\right] \\
& K_{i a}^{\prime}(x) \approx e^{-\pi a / 2}\left[\int_{\tau_{0}}^{\infty} e^{-\Psi(\tau)}(\cos \chi A(\tau)+\sin \chi C(\tau)) d \tau+\mathcal{O}\left(e^{-\pi a / 2}\right)\right] \\
& L_{i a}(x) \approx \frac{e^{\pi a / 2}}{2 \pi}\left[\int_{\tau_{0}}^{\infty} e^{-\Psi(\tau)}\left(\sin \chi-\cos \chi \frac{d \sigma}{d \tau}\right) d \tau+\mathcal{O}\left(e^{-\pi a / 2}\right)\right] \\
& L_{i a}^{\prime}(x) \approx \frac{e^{\pi a / 2}}{2 \pi}\left[\int_{\tau_{0}}^{\infty} e^{-\Psi(\tau)}(\sin \chi A(\tau)-\cos \chi C(\tau)) d \tau+\mathcal{O}\left(e^{-\pi a / 2}\right)\right]
\end{aligned}
$$

where

$$
\tau_{0}=\mu-\tanh \mu .
$$

These approximations can be used for moderately large $a$, which is the region where integrals for the oscillatory case are employed in the code [6].

It is however useful to have the complete expressions for testing the rest of the methods. The computation through quadrature using Eqs. (20), (24), (27), (29), $(32),(34)$ and $(35)$, provides a way for computing the functions in the whole $(x, a)$ plane, except close to $a=x$, where the integrands become non-smooth. For this reason, they have been used for checking the algorithm, although in the oscillatory region only Eq. (37) is necessary when building the numerical algorithm [6].

2.4.3 Quadrature rule. As reported in Goodwin [10], the trapezoidal rule is a very efficient method of computation of integrals $\int_{-\infty}^{+\infty} f(x) d x$ for rapidly decaying integrands $f(x)$; in particular, it is know that the error decays as $\exp \left(-(\pi / h)^{2}\right)$ for integrals of the type $\int_{-\infty}^{+\infty} e^{-x^{2}} g(x) d x$ with $g$ analytic in $\{z \in \mathbb{C}:|\Im z|<\pi / h\}$. After appropriate changes of variable, similar arguments follow for integrals over finite intervals with a smooth integrand $[13 ; 14 ; 15]$.

The semi-infinite integrals in this Section are appropriate for their computation by using the trapezoidal rule, because they decay as a double exponential as $\tau \rightarrow$ $+\infty$. On the other hand, the integrals over finite intervals show abrupt variations as $a \rightarrow x$, particularly in the oscillatory case, but under an adequate change of variables they can be also computed efficiently by means of the trapezoidal rule. For finite integrals, we consider a change of variable in order to map the finite interval $[a, b]$ into $(-\infty,+\infty)$ and a successive change to improve the convergence of the trapezoidal rule $[14 ; 15]$; namely, we consider the following transformation:

$$
\begin{aligned}
& I=\int_{a}^{b} f(x) d x=\int_{-\infty}^{+\infty} g(t) d t, g(t)=f(x(t)) \frac{(b-a) \cosh t}{2 \cosh ^{2}(\sinh t)}, \\
& x(t)=\frac{b+a}{2}+\frac{b-a}{2} \tanh (\sinh t) .
\end{aligned}
$$

And the integral is discretized by means of the trapezoidal rule with equal mesh 
size:

$$
\int_{-\infty}^{+\infty} g(t) d t=h \sum_{n=-\infty}^{+\infty} g(n h)+\epsilon,
$$

where the error $\epsilon$ is expected to decay very fast as the mesh size is decreased because the integrand is analytic and its decay is doubly exponential. We use a trapezoidal rule which halves the mesh size until the prescribed precision is reached; the same rule controls that the truncation of the infinite series (39) gives an error well below the accuracy claim.

Regarding the semi-infinite integrals, we use a change of variable to transform the integration interval $[a,+\infty)$ to $(-\infty, \infty)$. We consider the following change of variables to perform this map.

$$
\tau(x)=a+\sinh ^{-1}\left(e^{x}\right) .
$$

The additional change $x=\sinh t$ improves the convergence of the trapezoidal rule.

It is observed that, typically, no more than 8 iterations of the trapezoidal rule are needed, which means that the integrands are evaluated at $2^{8}+1=257$ points in the worst cases. This is the typical number of iterations for the evaluation of $\int_{-\infty}^{+\infty} e^{-x^{2}} d x$ by means of a recursive trapezoidal rule when double precision accuracy is demanded. This fact confirms that the above mentioned changes of variable are adequate for the computation of the integrals for the modified Bessel functions.

\subsection{Continued fraction method}

As discussed in [7] both $K_{i a}(x)$ and $K_{i a}^{\prime}$ can be computed for moderate $a$ by means of a continued fraction method, similar to the corresponding method for Bessel functions of real orders (see [16] and [12], pp. 239-240). We refer to [7] for a full description of this scheme.

As numerical experiments show, this method is competitive in speed with asymptotic expansions for large $x$ (Section 2.2) and the range of application is larger. Therefore, the continued fraction method substitutes the use of asymptotic expansions for large $x$.

\section{RANGE OF COMPUTATION AND SCALED FUNCTIONS}

As described in previous sections, the integral representations which were developed indicate that the dominant behaviour for the functions when the parameters are large is of exponential type. This means that the computations can only be carried for not too large values of $a$ and $x$ in order to avoid overflows/underflows in the computation. For instance, from Eqs. (29) and (32) it is seen that for large $a$ $(a>x)$, we have $K_{i a}(x) \sim e^{-a \pi / 2}$ and similarly for the derivative, while for $L_{i a}(x)$ and its derivative (Eqs. (34) and (35)) the asymptotic behaviour is $\sim e^{a \pi / 2}$. This means that to avoid overflow/underflows in the computation, we must restrict the range of $a$ in the oscillatory region to

$$
a<2 \ln \left(10^{n} N\right) / \pi
$$

where $N$ is either the inverse of the underflow number (when computing $K_{i a}(x)$ or its derivative) or the overflow number (for $L_{i a}(x)$ and its derivative); $10^{n}$ is a 
safety factor (in the program, we take $n=8$ ). For processors using the IEEE standard in double precision this will approximately limit $a$ to $a<440$. On the other hand, for the monotonic region $(x>a)$ the integral representations show that the dominant exponential behaviour is $K_{i a}(x) \sim e^{-\lambda}, L_{i a}(x) \sim e^{+\lambda}$ where $\lambda(x, a)=$ $x(\cos \theta+\theta \sin \theta), \sin \theta=a / x(\theta \in[0, \pi / 2])$, and similarly for the derivatives. This means that, in order to avoid overflows/underflows, the range of computation must be restricted to:

$$
\lambda(a, x)=\sqrt{x^{2}-a^{2}}+a \arcsin (a / x)<\ln \left(10^{n} N\right) .
$$

Figure 1 shows the computable range for $10^{n} N=10^{300}$ (typical value for IEEE standard double precision)

Given that all our expressions have the dominant exponential contributions factored out, exponentially scaled functions can be defined which are computable in wider ranges. Namely, we define:

$$
\widetilde{K_{i a}}(x)=\left\{\begin{array}{l}
e^{\lambda(x, a)} K_{i a}(x) \quad x \geq a \\
e^{a \pi / 2} K_{i a}(x) \quad x<a
\end{array} \widetilde{K_{i a}^{\prime}}(x)=\left\{\begin{array}{l}
e^{\lambda(x, a)} K_{i a}^{\prime}(x) \quad x \geq a \\
e^{a \pi / 2} K_{i a}^{\prime}(x) \quad x<a
\end{array}\right.\right.
$$

and

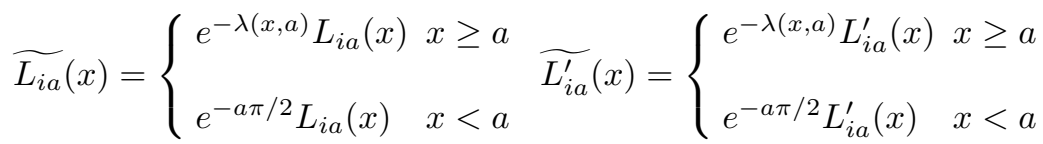

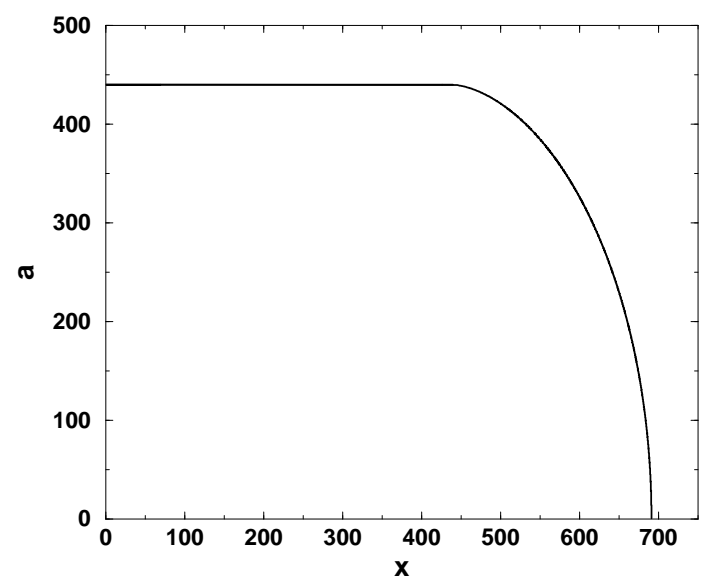

Figure 1. Computable range for the evaluation of $K_{i a}(x), L_{i a}(x)$ and their derivatives.

Note that, as in the rest of the article, we are considering positive $a$ because $K_{i a}(x)$ and $L_{i a}(x)$ are even functions of $a$. Of course, when applying the scaling factors for negative $a$, we should replace $a$ by $|a|$ in the exponential scaling factors of Eqs. (43) and (44). In this way, the scaled functions are also even functions of a.

The definitions in (43) and (44) eliminate exactly the front exponential factor in the oscillatory region $(a>x)$ from the series and the Airy type asymptotic 
expansion and in all the $(x, a)$ plane for the the integral representations. In other cases, there remains an exponential factor with soft variation. For example, when using Airy-type expansions in the monotonic region (neglecting non-exponential factors), we have

$$
\widetilde{K_{i a}}(x) \sim e^{\lambda-a \pi / 2}=e^{\widetilde{\lambda}}
$$

where

$$
\widetilde{\lambda}=x(\cos \theta+(\theta-\pi / 2) \sin \theta)=\sqrt{x^{2}-a^{2}}+a(\arcsin (a / x)-\pi / 2),
$$

which is small for $x \simeq a(\theta \simeq \pi / 2)$; loss of accuracy in the computation of $\tilde{\lambda}$ for $x \simeq a$ can be reduced by expanding $\tilde{\lambda}$ in powers of $\theta-\pi / 2$.

Similarly, an exponential factor remains when rescaling the asymptotic expansions and the same happens when applying the continued fraction method. In this case, we have for $x>a$;

$$
\widetilde{K_{i a}}(x) \sim e^{x-\lambda}=e^{-\bar{\lambda}}
$$

where

$$
\bar{\lambda}=x((\cos \theta-1)+\theta \sin \theta)=x-\sqrt{x^{2}-a^{2}}-a \arcsin (a / x),
$$

which goes to zero as $a / x \rightarrow 0(\theta \rightarrow 0)$. Loss of accuracy in the computation of $\bar{\lambda}$ for small $\theta$ ( $a / x$ small $)$ can be avoided by expanding $\bar{\lambda}$ in powers of $\theta$.

\section{ACKNOWLEDGMENTS}

A. Gil acknowledges financial support from Ministerio de Ciencia y Tecnología (Programa Ramón y Cajal). A. Gil and J. Segura acknowledge CWI Amsterdam for the hospitality and financial support.

\section{REFERENCES}

[1] M. Abramowitz, I. Stegun (Eds). Handbook of Mathematical Functions. National Bureau of Standards. Applied Mathematics Series, no. 55. U.S. Government Printing Office, Washington DC (1964).

[2] C.B. Balogh. Asymptotic expansions of the modified Bessel function of the third kind of imaginary order. SIAM J. Appl. Math. 15(5) (1967) 1315-1323.

[3] W.J. Cody, K.E. Hillstrom. Chebyshev approximations for the Coulomb phase shift. Math. Comp. 24 (1970) 671-677.

[4] T.M. Dunster. Bessel functions of purely imaginary order, with an application to second order linear differential equations having a large parameter. SIAM J. Math. Anal. 21(4) (1990) 95-1018.

[5] B. R. Fabijonas. Laplace's method on a computer algebra system with an application to the real valued modified Bessel functions. J. Comput. Appl. Math. 146 (2002) 323-342.

[6] A. Gil, J. Segura, N. M. Temme. Algorithm xxx: modified Bessel functions of imaginary order and positive argument. ACM Trans. Math. Soft. (submitted for publication in this same issue).

[7] A. Gil, J. Segura, N. M. Temme. Evaluation of the modified Bessel function of the third kind for imaginary orders. J. Comput. Phys. 175 (2002) 398-411

[8] A. Gil, J. Segura, N.M. Temme. Computation of the modified Bessel function of the third kind of imaginary orders: uniform Airy-type asymptotic expansion. J. Comput. Appl. Math. 153 (2003) 225-234. 
[9] A. Gil, J. Segura, N.M. Temme. Algorithm 819: AIZ,BIZ: Two Fortran 77 Routines for the Computation of Complex Airy Functions. ACM Trans. Math. Soft. 28 (2002) 325-336.

[10] E. T. Goodwin. The evaluation of integrals of the form $\int_{-\infty}^{\infty} f(x) e^{-x^{2}} d x$. Proc. Cambridge Philos. Soc. 45( 1949) 241-245.

[11] F.W.J. Olver. Asymptotics and Special Functions. Reprinted by A.K. Peters Ltd., 1997.

[12] W.H.Press, S.A. Teukolsky, W.T. Vetterling, B.P. Flannery. Numerical recipes in FORTRAN. The art of scientific computing. Second edition. Cambridge University Press, Cambridge, 1992.

[13] C. Schwarz. Numerical integration of analytic functions. J. Comput. Phys. 4 (1969) 19-29.

[14] H. Takahasi, M. Mori. Quadrature formulas obtained by variable transformation. Numer. Math. 21 (1973) 206-219.

[15] H. Takahasi, M. Mori. Double exponential formulas for numerical integration. Publ. Res. Inst. Math. Sci. 9 (1974) 721-741.

[16] N. M. Temme. On the numerical evaluation of the modified Bessel function of the third kind. J. Comput. Phys. 19(3) (1975), 324-337.

[17] N.M. Temme. Steepest descent paths for integrals defining the modified Bessel functions of imaginary order. Methods Appl. Anal. 1(1) (1994), 14-24.

[18] N.M. Temme. Numerical algorithms for uniform Airy-type asymptotic expansions. Numer. Algorithms 15(2) (1997) 207-225. 